\title{
Locally Advanced Disease Clinical Trial Setting
}

National Cancer Institute

\section{Source}

National Cancer Institute. Locally Advanced Disease Clinical Trial Setting. NCI Thesaurus. Code C124451.

A clinical trial or treatment setting in which the therapy of interest is used to treat locally advanced disease. 